\title{
Solitons and (spin-)Peierls transition in disordered quasi-one-dimensional systems
}

\author{
M. Mostovoy* and D. Khomskii ${ }^{\dagger}$ \\ University of Groningen, Nijenborgh 4, 9747 AG Groningen, The Netherlands
}

(October 25, 2018)

\begin{abstract}
We study the (spin-)Peierls transition in quasi-one-dimensional disordered systems, treating the lattice classically. The role of kinks, induced thermally and by disorder, is emphasized. For weak interchain interaction the kinks destroy the coherence between different chains at a temperature significantly lower than the mean-field Peierls transition temperature. We formulate the effective Ising model, which describes such a transition, investigate the doping dependence of the (spin-)Peierls transition temperature and discuss several implications of the picture developed. The results are compared with the properties of the spin-Peierls system $\mathrm{CuGeO}_{3}$.
\end{abstract}

PACS numbers: 64.70Kb, 75.10Jm

\section{INTRODUCTION}

The tendency towards the lattice instability is strongly enhanced in quasi-onedimensional systems. Conducting chains are inherently unstable against the periodic lattice distortion which opens a gap in the spectrum of electron excitations (Peierls instability). $\mathrm{TaS}_{3}, \mathrm{NbSe}_{3}, \mathrm{~K}_{0.3} \mathrm{MoO}_{3}$ are the examples of the quasi-one-dimensional conductors, which at a sufficiently low temperature become Peierls insulators. odic distortion was also observed in organic (TTF-CuBDT 3 , TTF-AuBDT Q $^{2}$, (MEM)$(\mathrm{TCNQ})_{2}$ 目, etc. $)$ and inorganic $\left(\mathrm{CuGeO}_{3} \mathrm{O}, \mathrm{NaV}_{2} \mathrm{O}_{5}\right.$ 暏 $)$ compounds, consisting of weakly interacting spin chains.

The excitation spectrum in the quasi-one-dimensional electronic and spin Peierls materials is usually interpreted in terms of the excitations in isolated chains. Nonetheless, the (spin-)Peierls transition is a three-dimensional phenomenon, because electronic or spin

\footnotetext{
*also at G. I. Budker Institute of Nuclear Physics, 630090 Novosibirsk, Russia.

$\dagger^{\dagger}$ also at P. N. Lebedev Physical Institute, Leninski prosp. 53, Moscow, Russia.
} 
chains are immersed in a crystalline lattice of a bulk material. In the standard description of the (spin-)Peierls transition the lattice is treated in the mean-field approximation. 12 In this approach, the excitations, which eventually destroy the ordered Peierls phase at a critical temperature, are the electron-hole (spin) excitations in isolated chains with a fixed lattice distortion.

It is well-known, however, that in these systems there exist also excitations of a different nature: solitons, or kinks, which are the mobile domain walls. In this contribution we consider the Peierls systems, in which the lattice in the ordered phase is dimerized, i.e. long and short bonds alternate. In this case the distortion wave is commensurate with lattice and cannot slide as a whole. On the other hand, kinks, separating domains with the opposite sign of the dimerization, can propagate along chains. Solitons are mixed electron-lattice (or spin-lattice) excitations. Their topological nature is responsible for the anomalous charge-to-spin ratio, which was used to explain the unusual bevavior upon doping of the electronic Peierls material trans-polyacetylene.13 For spin-Peierls systems, the soliton picture is much less popular, although it was discussed also in this context in Ref. 14 .

In addition to kinks, excited thermally or optically, there can be also kinks induced by disorder. Here we consider only one kind of disorder, which effectively cuts chains into finite segments. The interruption of chains may be caused by strong conformational disorder (e.g. bond bending in trans-polyacetylene), or by substitution of atoms in chains (as in $\mathrm{CuGeO}_{3}$ ). The minimal energy lattice configuration strongly depends on whether the number of atoms in a chain is even or odd: an odd chain contains one kink, 0 , 27 while an even chain does not. The disorder-induced kinks are neutral and have spin $\frac{1}{2}$. In fact, any kind of the off-diagonal disorder results in the appearance of kinks, 18 , 19 so the picture we consider here is rather general.

Although kinks provide a coherent explanation of a vast variety of experimental data on optical and magnetic properties of trans-polyacetylene, 13, 15 their role in the Peierls phase transition was not properly studied. That may be related to the fact, that transpolyacetylene has a large gap $E_{g} \approx 1.8 \mathrm{eV}$, so that the transition into the undimerized state cannot be observed. On the other hand, the critical temperature in spin-Peierls materials is rather low $(2-35 \mathrm{~K})$, and there exist a very detailed information on these transitions. An important property of the recently discovered inorganic spin-Peierls compound $\mathrm{CuGeO}_{3}$ is its ability to accept dopands, so that the disorder can be introduced into this system in a controlled way. This made possible the systematic study of the effect of disorder on the spin-Peierls transition.20 24 Finite chains in the spin-Peierls compound $\mathrm{CuGeO}_{3}$ are formed upon the substitution of $\mathrm{Cu}$ atoms in the $\mathrm{CuO}_{2}$ spin chains by nonmagnetic Zn, which interrupts the spin-exchange. Similar effect is reached by the 
substitution of Ge by Si.25 Doping by Zn or Si strongly decreases the Peierls transition

temperature and gives rise to the antiferromagnetic phase,2123 which coexists with the dimerization.21, 20 will show, that these effects can be naturally explained in terms of the disorder-induced kinks.

In this contribution we consider the (spin-)Peierls transition into a dimerized state in disordered quasi-one-dimensional systems, taking into account the soliton excitations. We believe that, due to their topological nature, kinks play an important role in the description of this transition, even though the interaction between the chains can considerably modify their properties. The approach we propose is, in a sense, opposite to the meanfield description of the (spin-)Peierls transition: we neglect the electron(spin) excitations, as well as the small fluctuations of the order parameter, and leave only kinks. In other words, we assume that locally the dimerization persists well above the critical temperature, and that the transition occurs when the coherence between the dimerization phases on different chains is established. The effective model, which describes such transition in clean systems, is the strongly anisotropic Ising model. We propose a simple modification of this model, which allows us to consider both thermally and disorder-induced kinks.

Our approach is valid if the coherence of the (spin-)Peierls state is destroyed by the kinks at a temperature significantly lower than the mean field transition temperature, so that the gap in the spectrum of the (spin) electron excitations is still large at the phase transition point and all nontopological excitations can be neglected. As we shall see, this requires a rather weak interchain interaction. Another important approximation we make, is the classical treatment of the soliton motion. The classical treatment of lattice is justified, if the value of the relevant phonon frequency is much smaller than the value of the Peierls gap. This is usually the case for the electronic Peierls materials (e.g. trans-polyacetylene). On the other hand, in spin-Peierls materials with a relatively small spin gap this would require a particularly soft lattice, which, in principle, can happen due to a proximity to some structural transition. 1 If, on the contrary, the Peierls gap is comparable to the optical phonon frequency, or is much smaller, then the consideration of the three-dimensional lattice has to be quantum.

\section{WEAKLY COUPLED FINITE CHAINS}

In this section we introduce a simple model of disordered quasi-one-dimensional (spin)Peierls material. There are three essential assumptions on which our model is based: (i) interchain interaction is assumed to be sufficiently weak; (ii) off-diagonal disorder is assumed to break effectively chains into segments; (iii) lattice can be treated classically.

Because of the weakness of the interchain interaction, in the first approximation chains 
can be considered independently of each other. The typical length of a segment in a disordered system is inversely proportional to the concentration of impurities. The classical lattice configuration of a finite chain has to be determined by minimizing the total chain energy, which includes the electron energy (the energy of the spin system for spin-Peierls materials), as well as the lattice energy. Below we discuss the form of the minimal energy lattice configuration of finite chains.

We begin by considering a conducting chain described by the Peierls-Hubbard (PH) Hamiltonian,

$$
\begin{aligned}
H_{P H}= & -\sum_{n, \sigma}\left(t_{0}+\alpha\left(u_{n}-u_{n+1}\right)\right)\left(c_{n \sigma}^{\dagger} c_{n+1 \sigma}+c_{n+1 \sigma}^{\dagger} c_{n \sigma}\right)+U \sum_{n} c_{n \uparrow}^{\dagger} c_{n \uparrow} c_{n \downarrow}^{\dagger} c_{n \downarrow} \\
& +\frac{K}{2} \sum_{n}\left(u_{n}-u_{n+1}\right)^{2},
\end{aligned}
$$

where the operator $c_{n \sigma}$ annihilates an electron with spin projection $\sigma$ at a site $n$, and $u_{n}$ is the shift of the $n$-th atom in the chain direction. The energy of a half-filled chain with even number of atoms and periodic boundary conditions is minimal when the chain is uniformly dimerized,

$$
u_{n}=(-)^{n} u_{0}
$$

which corresponds to alternation of long and short bonds. The dimerization takes place at all values of $U$. For non-interacting electrons $(U=0), 13,26$

$$
\frac{\alpha u_{0}}{t_{0}} \propto e^{-\frac{1}{\lambda_{e p}}}
$$

where $\lambda_{e p}$ is the dimensionless electron-phonon coupling constant,

$$
\lambda_{e p}=\frac{4 \alpha^{2}}{\pi t_{0} K} .
$$

In the opposite limit of strong electron correlations, $U \gg 4 t_{0}$, the value of the dimerization is determined by the interaction of the lattice with the low energy spin degrees of freedom, described by the spin-Peierls (SP) Hamiltonian,

$$
H_{s p}=\frac{1}{2} \sum_{n}\left(J_{0}+\alpha^{\prime}\left(u_{n}-u_{n+1}\right)\right)\left(\vec{S}_{n} \cdot \vec{S}_{n+1}\right)+\frac{K}{2} \sum_{n}\left(u_{n}-u_{n+1}\right)^{2},
$$

where

$$
J_{0}=\frac{4 t_{0}^{2}}{U}, \text { and } \alpha^{\prime}=\frac{8 t_{0}}{U} \alpha
$$

In this case 12

$$
\frac{\alpha u_{0}}{t_{0}} \propto\left(\frac{t_{0} \lambda_{e p}}{U}\right)^{\frac{3}{2}}
$$


The number of bonds in even chain with periodic boundary conditions (a ring) is also even, so that there are two degenerate minimal energy lattice configurations, shifted by one lattice constant with respect to each other. The amplitude of the distortion wave, $u_{0}$, for these two lattice configurations has opposite signs. The boundary conditions for finite chains produced by substitution of atoms are, however, free rather than periodic. For an open chain with even number of atoms there is only one minimal energy configuration, since the number of bonds in such chain is odd, so that the configuration, which has more short bonds, has lower energy (see Fig.1a). The value of the dimerization changes somewhat from the middle to the ends of the chain, but the sign of the dimerization is everywhere the same.

On the other hand, if the number of atoms in a chain is odd, the minimal energy lattice configuration has a form of a kink (see Fig.1b). The chain energy is practically independent of the position of the kink, provided that the distance from the kink to the nearest chain end is larger than the kink size. One unpaired spin is localized near the kink. Therefore, a spin $\frac{1}{2}$ object in an isolated odd segment, contrary to a common belief, is localized not close to an impurity (chain end), but rather away from it.

Next we consider the effects of interchain interaction. This interaction may originate, for instance, from a relatively small hopping of electrons between chains (which corresponds to the exchange between spins sitting on neighbouring chains in spin-Peierls materials), long range Coulomb interaction between the electrons and deformations of the three-dimensional lattice. Independently of the origin, the interchain interaction tends to create a coherence between the phases of the distortion waves on different chains. In a clean material it determines a type of crystalline structure. For simplicity we will assume, that the lattice configuration has the lowest energy when the sign of the dimerization is the same on all chains. (Actually, one can show that in $\mathrm{CuGeO}_{3}$ the distortions in neighbouring chains along $b$-axis have opposite phases, but it does not modify our conclusions.)

When a finite chain, created by disorder, is surrounded by other chains, its lattice configuration is different from that of an isolated chain. For instance, there appear two kinds of even chains: those in which the sign of the dimerization, favoured by the boundary conditions, is the same as the sign of the average dimerization in the bulk material, and those in which it is the opposite (see Fig.2a). In the latter case the energy loss due to interchain interaction is proportional to the chain length. Therefore, despite the weakness of interchain interaction it will be energetically more favourable in a sufficiently long chain to create a kink near one end of the chain and antikink near the opposite end, so that the sign of the dimerization between the domain walls is the same as the sign of the averaged dimerization (see Fig.2b). Similarly, the interchain interaction binds kink 
in an odd segment to one of the chain ends (see Fig.3). This, in a sense, restores the conventional picture of a spin $\frac{1}{2}$ sitting relatively close to impurity at an average distance of the order of a kink size $\xi$ (see also Ref. 25).

\section{QUALITATIVE DISCUSSION OF THE PHASE TRANSITION IN CLEAN AND DISORDERED SYSTEMS}

In this section we discuss qualitatively the (spin-) Peierls phase transition in quasione-dimensional systems with very weak interchain interaction. First we consider the clean systems. The long range order in an infinitely long isolated chain is destroyed by thermally excited kinks at any non-zero temperature. This happens because the energy of kink does not depend on its position in an isolated chain. In the presence of the interchain interaction the string with the opposite sign of the dimerization is created between the soliton and antisoliton. It costs energy proportional to the length of the string $l$, which results in a linear potential between kinks,

$$
V(l)=\lambda l
$$

The coefficient $\lambda$ measures the strength of the interchain interaction and may be called the string tension. The potential tends to bind solitons and antisolitons into pairs. The phase transition now occurs at a finite temperature, when the thermally excited solitonantisoliton pairs dissociate. This happens when the pair size,

$$
R(T)=\frac{T}{\lambda}
$$

becomes comparable to the distance between the thermally induced kinks in an isolated chain,

$$
d(T)=\exp \left(\frac{\mu}{T}\right)
$$

where $\mu$ is the kink excitation energy, which is of the order of gap in electron (spin) excitation spectrum. In this way for weak interchain coupling, $\lambda \ll \mu$, we obtain approximately

$$
T_{c}(0) \sim \frac{\mu}{\ln \frac{\mu}{\lambda}}
$$

In the doped Peierls system, apart from the thermally induced kinks, there exist also kinks induced by disorder, whose density is proportional to the concentration of dopands. The total density of kinks is 


$$
n_{\text {tot }}=n_{\text {therm }}+n_{\text {dis }}=e^{-\frac{\mu}{T}}+C x
$$

where $C$ is some numerical factor of the order of 1 . We can now estimate the phase transition temperature $T_{c}(x)$ in the same way as we did for a clean system, using $1 / n_{\text {tot }}$ as the average distance between the kinks. At small $x$, when the density of the disorderinduced kinks $n_{d i s}$ is much smaller than the density of the thermally excited kinks $n_{\text {therm }}$, the phase transition temperature decreases linearly with $x$,

$$
T_{c}(x)=T_{c}(0)(1-A x)
$$

and the coefficient $A$ is large,

$$
A \sim \frac{\mu}{\lambda\left(\ln \frac{\mu}{\lambda}\right)^{2}} .
$$

A more careful estimate of $A$ will be given in the next section. If, on the other hand, $n_{\text {dis }} \gg n_{\text {therm }}$, the phase transition temperature becomes inversely proportional to the concentration,

$$
T_{c}(x)=\frac{C}{x}
$$

The interpretation of the last equation is, that at the phase transition point the typical distance from kink to the nearest end of odd segment, $\sim T / \lambda$, becomes comparable with the average length of the segment $\sim 1 / x$. This result is not applicable at very large $x$ : when an average length of the segment becomes of the order of soliton size, $1 / x \sim \xi_{0}$, there will be no ordering even at $T=0$.

Because the density of the thermally induced kinks is exponentially small at $T \ll \mu$, the disorder-induced kinks begin to dominate at a very low doping concentration,

$$
x_{c} \sim \frac{\lambda}{\mu} \ln \left(\frac{\mu}{\lambda}\right)
$$

and the crossover between linear and $\frac{1}{x}$ decrease of the transition temperature is very sharp for $\lambda \ll \mu$.

\section{EFFECTIVE ISING MODEL}

The model qualitatively discussed above, which describes the statistical properties of kinks, induced thermally or by disorder in the ensemble of weakly interacting finite chains, can be formulated as an Ising-type model. To this end we divide chains into cells containing two bonds. The sequence of bonds in each cell can be either short-long 
or long-short (positive or negative dimerization), which is decribed by the Ising variable $\sigma= \pm 1$. The uniformly dimerized lattice corresponds to the ferromagnetic ordering of the Ising spins. Kinks are domain walls in Ising variables, i.e., $\sigma_{n}=-1$ for $n$ less than some $m$ and $\sigma_{n}=+1$ for $n \geq m$, or vice versa. In this model one neglects the actual size of the kink, which is reasonable as long as it is much smaller than the average distance between the kinks. The weak interaction between different chains we treat in the mean-field approximation.28 The energy of the chain containing $N$ Ising spins has the form,

$$
E_{N}^{\nu \nu^{\prime}}=\frac{\mu}{2} \sum_{n=1}^{N}\left(1-\sigma_{n} \sigma_{n+1}\right)-h \sum_{n=1}^{N} \sigma_{n}-\frac{\mu^{\prime}}{2}\left(\nu \sigma_{1}+\nu^{\prime} \sigma_{N}\right)+\mu^{\prime}
$$

Here the first term describes the energy cost of a kink (equal to $\mu$ ), while the second term describes the interaction with the mean field $h$ of neighbouring chains. The third term is introduced to model different kinds of finite chains in (spin-)Peierls material. It has a form of the interaction with the "magnetic field" $h_{L}=\nu \mu^{\prime}$, applied at the left end, and $h_{R}=\nu^{\prime} \mu^{\prime}$ at the right end. Here $\nu$ and $\nu^{\prime}$ take values \pm 1 , which corresponds to four kinds of finite (spin-)Peierls chains: even segments in phase $\left(\nu=\nu^{\prime}=+1\right)$ and out of phase $\left(\nu=\nu^{\prime}=-1\right)$ with an average dimerization, and odd segments with one end in phase and other out of phase $\left(\nu=+1, \nu^{\prime}=-1\right.$ or vice versa). In the latter two cases, the opposite orientation of the magnetic field at the chain ends creates a kink in the lowest energy configuration of the Ising chain, provided that $\mu^{\prime}>\mu$. In what follows, we will restrict ourselves to the case of infinite $\mu^{\prime}$.

The partition function of the Ising chain can be easily found by means of the transfer matrix method.27 For $\nu^{\prime}=\nu$ the result is,

$$
Z_{N}^{\nu \nu}=\frac{e^{-\nu \bar{h}}}{2}\left[\left(\lambda_{+}^{N+1}+\lambda_{-}^{N+1}\right)+\nu \sin \psi\left(\lambda_{+}^{N+1}-\lambda_{-}^{N+1}\right)\right],
$$

while for $\nu^{\prime}=-\nu$ one has,

$$
Z_{N}^{+-}=Z_{N}^{-+}=\frac{\cos \psi}{2}\left(\lambda_{+}^{N+1}-\lambda_{-}^{N+1}\right)
$$

Here we denoted by $\lambda_{ \pm}$the eigenvalues of the transfer matrix,

$$
\lambda_{ \pm}=\cosh \bar{h} \pm \sqrt{(\sinh \bar{h})^{2}+e^{-2 \bar{\mu}}}
$$

and $\psi$ is defined by,

$$
\sin \psi=\frac{\sinh \bar{h}}{\sqrt{(\sinh \bar{h})^{2}+e^{-2 \bar{\mu}}}}
$$


Above we used the notations: $\bar{h}=\beta h$ and $\bar{\mu}=\beta \mu$.

The averaging over disorder reduces in our model to the averaging over $N, \nu$, and $\nu^{\prime}$. For simplicity we assume that for each $N$ all the four types of finite chains are possible. The distribution of the chain lengths $P_{N}$ for small concentration $x$ of randomly positioned dopands (e.g. $\mathrm{Zn}$ in $\mathrm{CuGeO}_{3}$ ) is

$$
P_{N}=\frac{1}{\langle N\rangle} \exp \left(-\frac{N}{\langle N\rangle}\right)
$$

where the average chain length is related to the concentration of dopands by,

$$
\langle N\rangle=\frac{2}{x},
$$

(factor 2 appears because the length of the unit cell in the effective Ising model is equal to $2 a$ ). For small concentration $x$ one can substitute the summation over $N$ by the integration.

The averaged free energy per unit cell is

$$
\langle f\rangle=\frac{1}{4\langle N\rangle} \sum_{N, \nu, \nu^{\prime}} P_{N} F_{N}^{\nu \nu^{\prime}}
$$

where $F_{N}^{\nu \nu^{\prime}}=-T \ln Z_{N}^{\nu \nu^{\prime}}$ is the free energy of a finite chain. The mean-field value $h$ is proportional to the averaged value of the Ising spin (the average dimerization),

$$
h=\lambda\langle\sigma\rangle=-\lambda \frac{\partial\langle f\rangle}{\partial h},
$$

where $\lambda$ is the coupling constant.

The transition temperature, $T=T_{c}$, is obtained by soving equation 28

$$
-\lambda \frac{\partial^{2}\langle f\rangle}{\partial h^{2}}(h=0)=1
$$

Evaluation of the second derivative of the average free energy gives

$$
\frac{\lambda e^{\bar{\mu}}}{T_{c}}\left[\frac{\gamma^{2}}{2} \zeta\left(2, \frac{\gamma}{2}\right)-1-2 \epsilon \gamma+4 \epsilon \gamma^{3}\left(\beta(\gamma)-\frac{1}{2 \gamma}\right)\right]=1
$$

where $\zeta(z, q)$ is the Riemann zeta function, 29

$$
\zeta(z, q)=\sum_{n=0}^{\infty} \frac{1}{(n+q)^{z}}
$$

and $\beta(q)$ is, 30

$$
\beta(q)=\sum_{n=0}^{\infty} \frac{(-)^{n}}{n+q}
$$


We also introduced the dimensionless parameter

$$
\gamma=x e^{\bar{\mu}},
$$

which is, roughly speaking, the ratio of the densities of the disorder-induced and the thermally-induced kinks, and

$$
\epsilon=\frac{1}{2} e^{\bar{\mu}} \ln \left(\operatorname{coth} \frac{\bar{\mu}}{2}\right) .
$$

For $T \ll \mu \epsilon$ is close to 1 .

If now $\gamma \ll 1$, i.e. the average distance between the thermally induced kinks is much smaller than the average chain size, the equation for $T_{c}(x)$ can be approximately written as follows:

$$
\frac{\lambda \exp \left(\frac{\mu}{T_{c}(x)}\right)}{T_{c}(x)}\left(1-2 \epsilon x \exp \left(\frac{\mu}{T_{c}(x)}\right)\right)=1 .
$$

At $x=0$ the critical temperature is determined by the equation,

$$
\exp \left(\frac{\mu}{T_{c}(0)}\right)=\frac{T_{c}(0)}{\lambda},
$$

which coincides exactly with the condition $R\left(T_{c}(0)\right)=d\left(T_{c}(0)\right)$, which we used in the previous Section (see Eqs. (91), (10), and (11)). The magnitude of the linear slope of $T_{c}(x)$-curve at $x=0$ (cf. Eqs.(13) and (14)) is

$$
A=-\frac{\frac{d T_{c}}{d x}(0)}{T_{c}(0)}=\frac{2 \epsilon T_{c}(0)}{\lambda \ln \left(\frac{e T_{c}(0)}{\lambda}\right)} .
$$

In the opposite limit $\gamma \gg 1$, when the disorder-induced solitons dominate, we obtain from Eq.(27),

$$
T_{c}(x)=\frac{\lambda}{6 x},
$$

which has to be compared with Eq.(15) of the previous Section.

Eq.(27) was solved numerically for the concentration of dopands $x \leq 0.08$ and several values $\lambda$. The results are presented in Fig. 4. The value of $\mu$ for each $\lambda$ was chosen to maintain $T_{c}(0)=14 K$. We see that the crossover between the linear and $\frac{1}{x}$ behavior of $T_{c}(x)$ is indeed very sharp.

\section{CONCLUSIONS}

The treatment carried above shows the important role played by solitons in describing properties of both clean and doped (spin-)Peierls systems. For the electronic Peierls material like polyacetilene, there are already a lot of experimental evidences of the existence 
of kinks (although due to a large kink energy they do not contribute significantly to the thermodynamic properties of this material at room temperature).

The mean-field soliton lattice solution was used to describe the thermodynamics of the incommensurate phase of spin-Peierls systems in strong magnetic field 11, 31 33 We have shown, however, that solitons could strongly affect the properties of spin-Peierls systems, both clean and doped, also at $H=0$. The small parameter $\lambda / \mu$ (where $\lambda$ is the strength of the interchain interaction and $\mu$ is the kink energy of the order of spin gap) is responsible for the sharp suppression of $T_{c}$ by doping (cf. Eqs.(14) and (34)). Qualitative estimates show, that the SP phase is completely suppressed, when the average distance between impurities becomes of the order of the coherence length of the SP ordering $\xi$,

$$
\xi \sim \frac{J_{0}}{E_{g}} a,
$$

or, in other words, when the average segment length becomes of the order of the kink size. For $\mathrm{CuGeO}_{3}, \xi \sim 8-10$ lattice spacings, so that the critical concentration for the complete suppression of SP transition $x_{c}$ cannot, in any case, exceed $\sim 10-12 \%$. Experiments give a somewhat smaller value: $x_{c} \sim 7-8 \%$ for $\mathrm{Zn}$ and $x_{c} \sim 2-3 \%$ for Si. The extra "efficiency" of Si, as compared to Zn, is, most probably, related to the fact that $\mathrm{Si}$, substituting $\mathrm{Ge}$, is located between two $\mathrm{CuO}_{2}$-chains and thus influences two chains simultaneously. Therefore, two kinks are created per one Si and only one kink per Zn. Correspondingly, Si doping may be expected to be two times more efficient in suppressing $T_{c}$ than the doping by $\mathrm{Zn}$.

An additional argument in favour of solitons induced in doped (spin-)Peierls systems, comes from certain analogy between the properties of doped $\mathrm{CuGeO}_{3}$ and the behaviour of this material at strong magnetic field 34 (in which case the soliton picture was checked experimentally $32 \sqrt[33]{3}$ ). The notion of solitons can, therefore, provide a unified approach to this problems.

Yet another problem, appearing in the discussion of the doped spin-Peierls systems, is the interplay between the SP and antiferromagnetic ordering. As was first observed in Refs. 21 and 24, the two phases coexist in some interval of the concentration of dopands. Soliton picture provides a natural explanation of this phenomenon 25 As was discussed above, the kinks created by doping carry spin $\frac{1}{2}$. They induce in their vicinitiy the antiferromagnetic correlations, which decay at the length scale $\xi$ along the chain (see Fig.5). The antiferromagnetic microregions, created in this way, interact with each other, giving finally the long-range magnetic order, but with a reduced sublattice magnetization, spatially varying due to disorder. It is important that the spins of the separated kinks are free, so that they can ajust to the mean field created by other spins. In other words, the disorder, inducing unpaired spins, does not introduce frustration in the magnetic 
interaction. Once the coherence between the spins is established, the Neel temperature, $T_{N}$, should grow, roughly speaking, linearly with the density of the disorder-induced spins, i.e., $T_{N} \propto x$ at small $x$. The detailed treatment of the interplay of the SP and antiferromagnetic ordering will be given in a separate publication.

A similar picture, but without an apparent use of the soliton concept, in which the local antiferromagnetic correlations are created in the regions where the lattice ajusts to impurities, was suggested in Ref. 35. Creation of free spins by nonmagnetic impurities in the rigidly dimerized chains was considered in Ref. 36.

Our model is, of course, oversimplified, as we assumed that the phase transition into disordered state occurs entirely due to the loss of coherence between the phases of the order parameter on different chains. In practice, the conventional spin and phonon excitations have to be included to get a reasonable description of the excitation spectrum, magnetic susceptibility and other properties of spin-Peierls materials. The aim of this contribution, is, however, to show that the large fluctuations of the order parameter (kinks), which are neglected in the mean-filed approach, can be important in the quasione-dimensional systems.

Summarizing, we can say that the concept of solitons, which is well established for the electronic Peierls materials, is also very useful for the discussion of spin-Peierls sys-

tems. It gives a natural framework for the description of the properties of disordered Peierls systems and allows one to explain the dependence of the spin-Peierls transition temperature on doping, the interplay of the spin-Peierls and antiferromagnetic ordering, the simultaneous influence of doping and magnetic field, etc.

\section{ACKNOWLEDGMENTS}

The authors are grateful to B. Büchner, M. T. Figge, W. Geertsma, J. Knoester and G. Sawatzky for useful discussions. This work was supported by the Dutch Foundation for Fundamental Research of Matter (FOM).

${ }^{1}$ R. E. Peierls, "Quantum Theory of Solids" (Clarendon, Oxford, 1995).

${ }^{2}$ D. W. Bullett in "Theoretical aspects of band structure and electronic properties of pseudoone-dimensional solids", ed. H. Kamimura, (Reidel, Dordrecht, 1985), p. 163. 
${ }^{3}$ J. W. Bray et al., Phys. Rev. Lett. 35, 744 (1975).

${ }^{4}$ I. S. Jacobs et al., Phys. Rev. B 14, 3036 (1976).

${ }^{5}$ S. Huisinga et al., Phys. Rev. B 19, 4723 (1979).

${ }^{6}$ M. Hase, I. Terasaki, and K. Uchinokura, Phys. Rev. Lett. 70, 3651 (1993).

${ }^{7}$ M. Isobe and Y. Ueda, J. Phys. Soc. Jpn, 65, 1178 (1965).

${ }^{8}$ Y. Fujii et al., to be published.

${ }^{9}$ M. J. Rice and S. Strässler, Solid State Commun. 13, 125 (1973).

${ }^{10}$ E. Pytte, Phys. Rev. B 10, 4637 (1974).

${ }^{11}$ L. N. Bulaevskii, A. I. Buzdin, and D. I. Khomskii, Solid State Commun. 27, 5 (1978).

12 M. C. Cross and D. S. Fisher, Phys. Rev. B 19, 402 (1979); M. C. Cross, Phys. Rev. B 20, 4606 (1979).

13 A. J. Heeger, S. Kivelson, J. R. Schrieffer, and W. P. Su, Rev. Mod. Phys. 60, 781 (1988).

14 T. Nakano and H. Fukuyama, J. Phys. Soc. Jpn., 49, 1679 (1980); 50, 2489 (1981).

${ }^{15}$ see for a review, "Conjugated Conducting Polymers", ed. H. Kies, (Springer, Berlin, 1992).

${ }^{16}$ W. P. Su, Solid State Commun. 35, 899 (1980).

${ }^{17}$ D. K. Campbell, T. A. DeGrand, S. Mazumdar, Phys. Rev. Lett. 52, 1717 (1984).

${ }^{18}$ K. Iwano et al., J. Phys. Soc. Japan, 58, 4048 (1989).

${ }^{19}$ M. V. Mostovoy, M. T. Figge, and J. Knoester, to be published.

${ }^{20}$ M. Hase, Y. Sasago, K. Uchinokura, and H. Obara, Phys. Rev. Lett. 71, 4059 (1993).

${ }^{21}$ S. B. Oseroff, S.-W. Cheong, B. Akitas, M. F. Hundley, Z. Fisk, and L. W. Rupp, Phys. Rev. Lett., 741450 (1995).

22 J. G. Lussier, S. M. Coad, D. K. McMorrow, and D. McK. Paul, J. Phys. Condens. Matter 
7, L325 (1995).

23 J. P. Renard, K. Le Dang, P. Veillet, G. Dhalenne, A. Revcolevschi, and L. P. Regnault, Europhys. Lett. 30, 475 (1995).

${ }^{24}$ L. P. Regnault, J. P. Renard, G. Dhalenne, and A. Revcolevschi, Europhys. Lett. 32, 579 (1995).

${ }^{25}$ D. Khomskii, W. Geertsma, and M. Mostovoy, Czech. Journ. of Phys., 46 Suppl. S6, 32 (1996).

${ }^{26}$ H. Takayama, Y. R. Lin-Liu, and K. Maki, Phys. Rev. 21, 2388 (1980).

27 J. B. Kogut, Rev. Mod. Phys., 51, 659 (1979).

${ }^{28}$ Y. Imry, P. Pincus, and D. Scalapino, Phys. Rev. B, 121978 (1975).

${ }^{29}$ I. S. Gradshteyn and I. M. Ryzhik, "Table of Integrals, Series and Products", (Academic Press, New York, 1994), p. 1100.

30 ibid., p. 956.

${ }^{31}$ M. Fujita and K. Machida, J. Phys. Soc. Jpn., 53, 4395 (1984).

32 T. W. Hijmans, H. B. Brom, and L. J. de Jongh, Phys. Rev. Lett. 54, 1714 (1985).

${ }^{33}$ G. Remenyi et al., Czech. Journ. of Phys., 46 Suppl. S4, 1959 (1996).

${ }^{34}$ B. Büchner, private communication.

${ }^{35}$ H. Fukuyama, T. Tanimoto, and M. Saito, J. Phys. Soc. Jpn. 65, 1183 (1996).

${ }^{36}$ G. B. Martins, E. Dagotto, and J. A. Riera, Phys. Rev. B, 5416032 (1996). 


\section{FIGURE CAPTIONS}

FIG.1. Schematic view of the minimal energy lattice configuration of an isolated finite (spin-)Peierls chain with even (a) and odd (b) number of atoms (double and single lines correspond, respectively, to short and long bonds). The order parameter (thin line) changes sign in odd chain, and one unpaired spin (indicated by an arrow) is located near the kink.

FIG.2. Finite segment (thick lines) with even number of sites out of phase with the surrounding chains (thin lines) creates a string of unfavourable phase (a). The string can be removed by creating a kink and antikink near the chain ends (b).

FIG.3. Kink in an odd segment moved to one of the chain ends to minimize the interchain interaction energy.

FIG.4. Dependence of the (spin-)Peierls transition temperature on concentration of dopands for $\lambda=3,2,1,0.5,0.1 \mathrm{~K}$ (the curves, respectively, (a) - (e)).

FIG.5. Antiferromagnetic correlations induced in the vicinity of kink. 


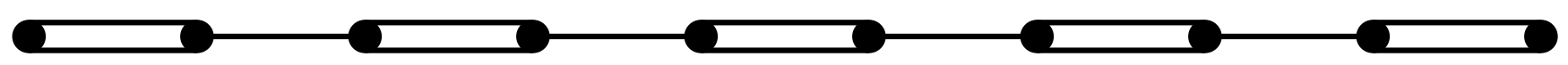

(a)

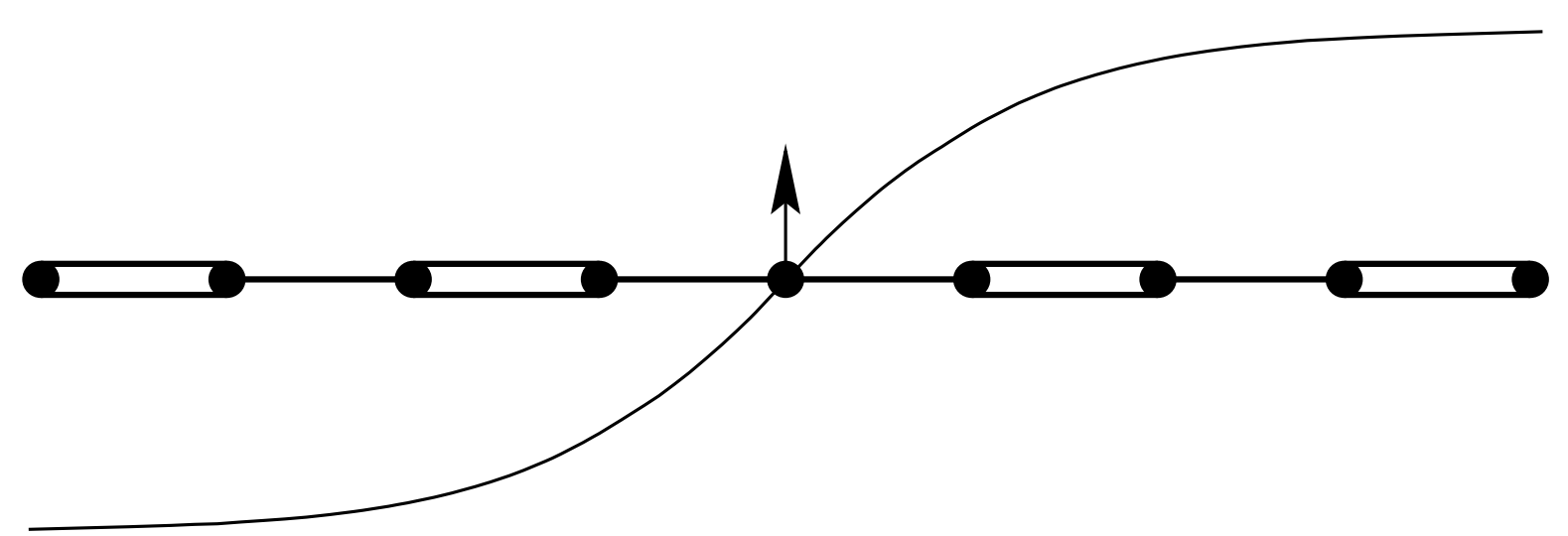

(b)

FIG.1. 

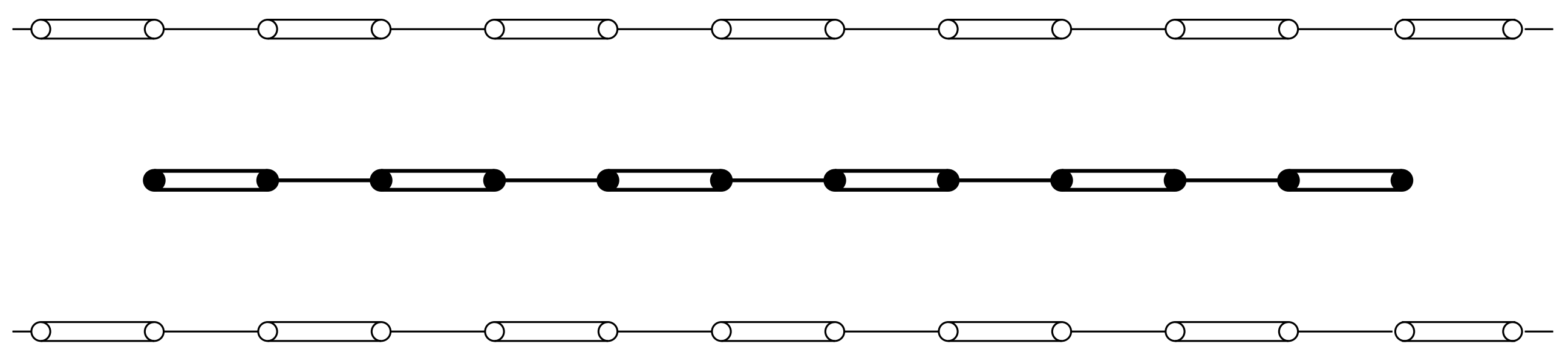

(a)
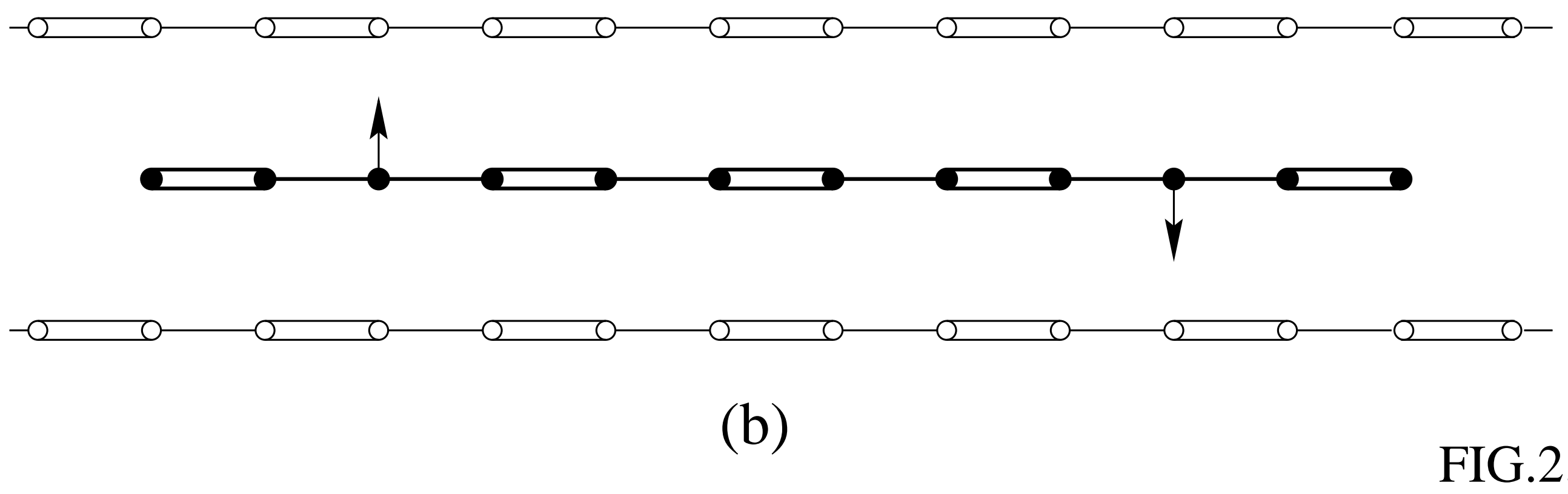

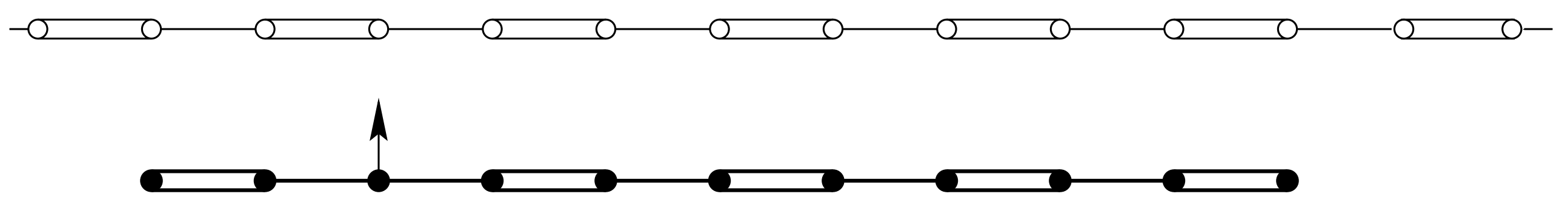

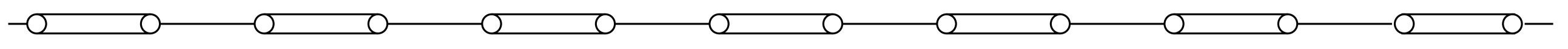

FIG.3 


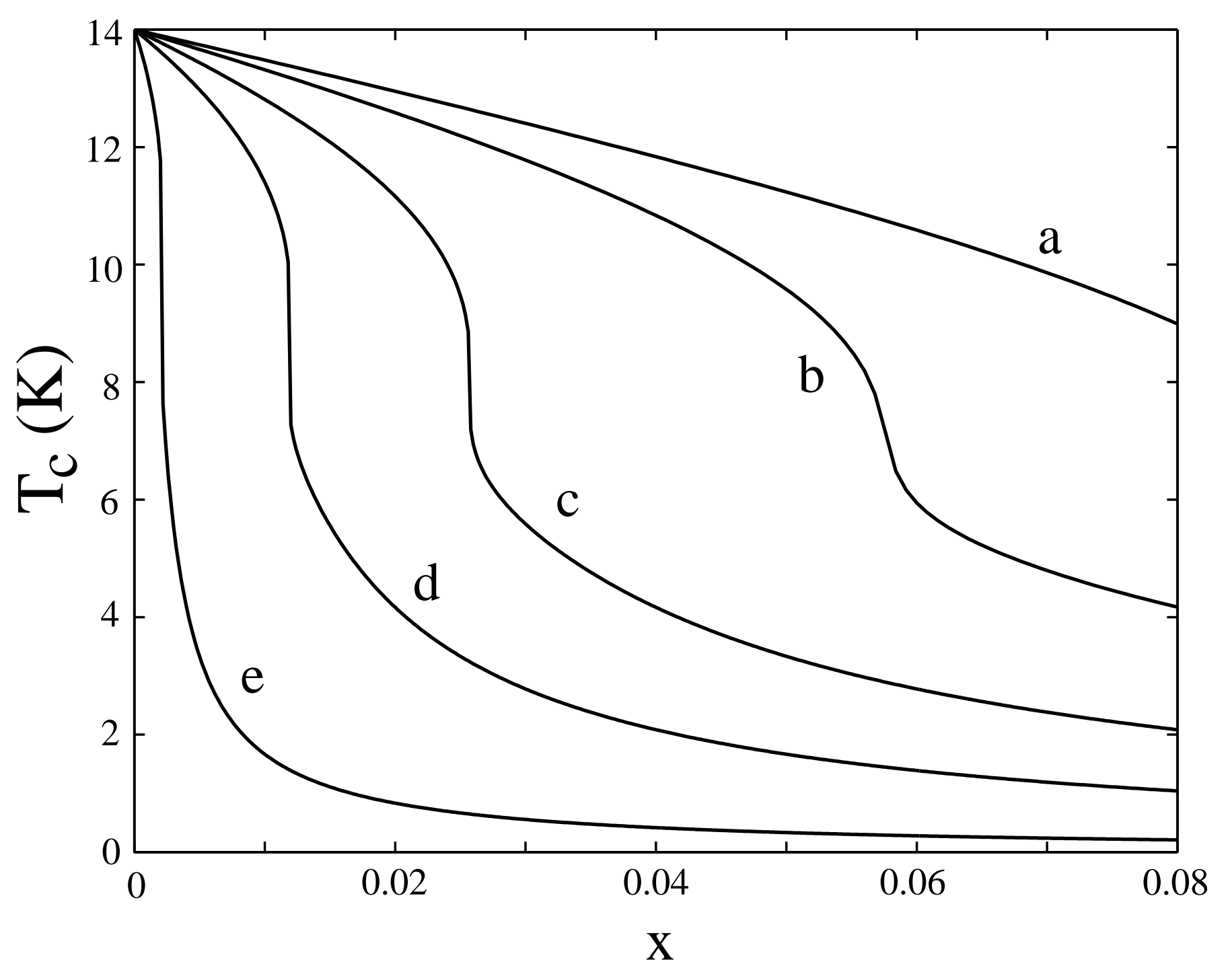

FIG.4. 


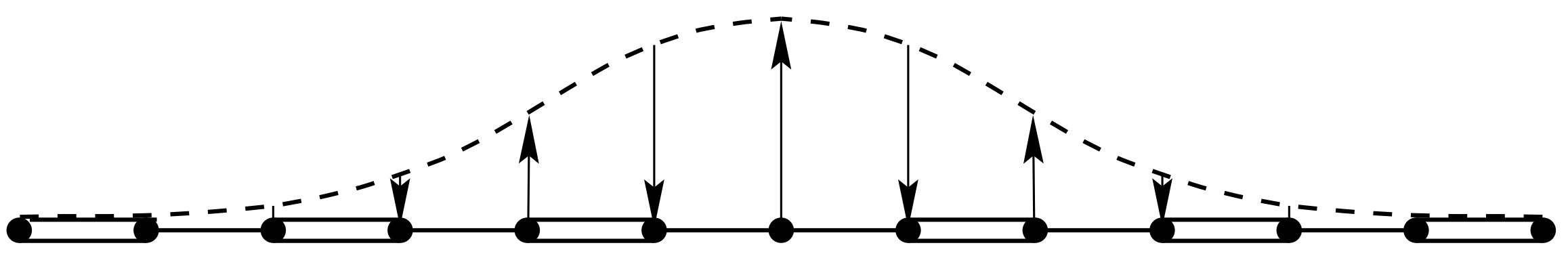

FIG.5. 